\title{
Suboptimal chemotherapy is an adverse prognostic factor in osteosarcoma
}

\author{
Bicheng Yong ${ }^{\dagger}$, Pingxian Tan ${ }^{\dagger}$, Junqiang Yin, Changye Zou, Xianbiao Xie, Jin Wang, Gang Huang, \\ Qianyong Wang and Jingnan Shen*
}

\begin{abstract}
Background: We sought to determine whether suboptimal chemotherapy compromised the prognosis of osteosarcoma patients.

Methods: A total of 132 eligible patients who underwent chemotherapy between 1998 and 2008 were identified in our database. Information regarding patient demographics, clinical characteristics, and survival status were extracted for analysis. Optimal chemotherapy was defined as receipt of $\geq 80 \%$ of the planned dose intensity of prescribed agents within the planned durations.

Results: The use of optimal chemotherapy resulted in an overall survival benefit with $P=0.006$. Patients who failed to complete the optimal chemotherapy protocol had a dismal prognosis of $30.8 \%$ overall survival over five years, whereas those who completed the optimal chemotherapy had an overall survival rate over five years of $65.3 \%$. Based on multivariate analysis, patients who were treated with a suboptimal protocol had a higher risk of relapse, metastasis and mortality. The hazard ratio (HR) of recurrence or death for the suboptimal chemotherapy group was as high as 2.512 over that of the optimal chemotherapy group ( $H R=2.512,95 \%$ confidence interval $=1.242$ to 3.729).
\end{abstract}

Conclusions: Chemotherapy is a significant independent prognostic variable, and suboptimal chemotherapy was found to have a detrimental effect on the outcome of patients with osteosarcoma.

Keywords: Osteosarcoma, Suboptimal chemotherapy, Survival

\section{Background}

Osteosarcoma is the most common malignant bone tumor; it predominantly affects adolescents and continues to be an extremely aggressive disease despite the use of neoadjuvant chemotherapy protocols and widemargin, limb-salvaging surgery [1]. The five-year overall survival (OS) rate of osteosarcoma patients of all ages has been reported to range from $53 \%$ to $58 \%$ in the United States and Europe [2].

An approximately $70 \%$ long-term event-free survival rate for osteosarcoma patients can currently be achieved by using the standard three-drug chemotherapy protocol that includes cisplatin, doxorubicin and high-dose methotrexate [3]. The addition of ifosfamide to a three-drug regimen has

\footnotetext{
* Correspondence: shenjingnan@126.com

†Equal contributors

Musculoskeletal Oncology Department, First Affiliated Hospital of Sun YatSen University, 14th floor \#58 Zhongshan 2nd Road, Guangzhou, Guangdong 510080, China
}

\section{() Biomed Central}

(c) 2012 Yong et al.; licensee BioMed Central Ltd. This is an Open Access article distributed under the terms of the Creative Commons Attribution License (http://creativecommons.org/licenses/by/2.0), which permits unrestricted use, distribution, and reproduction in any medium, provided the original work is properly cited. been extensively used and tested in several clinical trials [4-7]. In China, chemotherapy for osteosarcoma has lagged significantly behind other countries. Most chemotherapy treatments for osteosarcoma were adopted from other countries [8]. However, the phenomenon that patients often abandon treatment prematurely or choose to delay resuming chemotherapy is quite prevalent but is not seriously recognized in musculoskeletal oncology.

The present study aimed to evaluate the effect of incomplete or suboptimal chemotherapy on survival and to discuss possible reasons for lack of compliance to treatment. We found that suboptimal chemotherapy is an unfavorable prognostic factor in the overall survival (OS), event free survival (EFS), relapse free survival and metastasis free survival of osteosarcoma patients. Inadequate finances, poor patient-doctor relationship and psychological and behavioral compromise may contribute to suboptimal chemotherapy. 


\section{Methods}

Patient eligibility

We retrospectively reviewed the medical charts of 410 osteosarcoma patients who were treated at our hospital between 1998 and 2008. The last follow-up for eventfree survivors was in October, 2010. The minimum follow-up time was 24 months. The criteria for inclusion in the study were as follows: (1) Enneking stage IIB [9]; (2) no history of treatment except needle biopsy; (3) scheduled for neo- and adjuvant chemotherapy; (4) surgery at our institute; and (5) longer than 24 months of follow-up for event-free patients. Of the 410 patients, 367 were Enneking stage IIB. Patients were excluded for the following reasons: prior chemotherapy, surgery or open biopsy at another hospital $(\mathrm{n}=32)$, no treatment $(n=23)$, chemotherapy alone $(n=63)$, or surgery alone $(\mathrm{n}=9)$. In addition, patients who were followed for less than 24 months $(n=108)$ were excluded. Therefore, the final study population consisted of 132 patients; the clinicopathological characteristics of these patients are summarized in Table 1. Several variables were recorded, and the influence of the variables on survival, local recurrence, and metastasis were statistically evaluated. This study was approved by the Ethical Committee of First Affiliated Hospital, Sun Yat-Sen University.

\section{Protocol description}

Optimal chemotherapy was defined as follows: (1) the administration of methotrexate (MTX), cisplatin (DDP), adriamycin (ADM), ifosfamide (IFO) or their combinations; (2) the administration of MTX at a dose of 8 to 12 $\mathrm{g} / \mathrm{m}^{2}$ (with leucovorin'rescue' commencing six hours after the initiation of the MTX infusion), cisplatin at a dose of $100 \mathrm{mg} / \mathrm{m}^{2}$ for four hours followed by $60 \mathrm{mg} / \mathrm{m}^{2}$ of adriamycin for 48 hours, and IFO at a dose of 2 to $3 \mathrm{~g} / \mathrm{m}^{2}$ for five days with an injection of mesna (400 mg) after 12 hours of IFO administration; (3) one cycle of chemotherapy (MTX, DDP + ADM, IFO) that took place over 42 days; and (4) patients who received $\geq 80 \%$ of the planned dose intensity [10]. At least two courses of MTX were required for induction chemotherapy, and at least three cycles of triplets (MTX, DDP + ADM, IFO) were applied after definitive surgical therapy (Figure 1A). At our institute, IFO was introduced into our optimal protocol in 1999. Prior to that, postoperative chemotherapeutic regimens were the same as the preoperative regimens; after the inclusion of IFO, poor responders were administered IFO-containing regimens more frequently, while good responders were treated with the same regimen as for the preoperative therapy. The chemotherapy response was primarily determined based on physical evaluation and radiographic reassessment according to Response Evaluation Criteria in Solid Tumors (RECIST) criteria. A lesion in the same plane was consistently measured [11].
Table 1 Demographic and clinical characteristics of the cohort

\begin{tabular}{|c|c|c|}
\hline & Number & Percent of total $(100 \%)$ \\
\hline Total patients & 132 & 100 \\
\hline \multicolumn{3}{|l|}{ Age } \\
\hline$\leq 11(\mathrm{~F}), \leq 12(\mathrm{M})$ & 24 & 18.2 \\
\hline 12 to $14(\mathrm{~F}), 13$ to $15(\mathrm{M})$ & 34 & 25.8 \\
\hline 15 to $39(\mathrm{~F}), 16$ to $39(\mathrm{M})$ & 70 & 53.0 \\
\hline$\geq 40$ & 4 & 3.0 \\
\hline \multicolumn{3}{|l|}{ Gender } \\
\hline Male & 93 & 70.5 \\
\hline Female & 39 & 29.5 \\
\hline \multicolumn{3}{|l|}{ Location } \\
\hline Distal femur & 66 & 50.0 \\
\hline Proximal tibia & 35 & 26.5 \\
\hline Proximal humerus & 6 & 4.5 \\
\hline Proximal fibula & 11 & 8.3 \\
\hline Proximal femur & 4 & 3.0 \\
\hline Hip/pelvic & 3 & 2.3 \\
\hline Others & 7 & 5.3 \\
\hline \multicolumn{3}{|l|}{ Pathological fracture } \\
\hline Yes & 7 & 5.3 \\
\hline No & 125 & 94.7 \\
\hline \multicolumn{3}{|l|}{ Subtype } \\
\hline Osteoblast & 103 & 78.0 \\
\hline Chondroblastic & 13 & 9.8 \\
\hline Fibroblastic & 6 & 4.5 \\
\hline Others & 10 & 7.6 \\
\hline \multicolumn{3}{|l|}{ Chemotherapy } \\
\hline Optimal chemotherapy & 52 & 39.4 \\
\hline Suboptimal chemotherapy & 80 & 60.6 \\
\hline \multicolumn{3}{|l|}{ Local recurrence } \\
\hline Yes & 27 & 20.5 \\
\hline No & 105 & 79.5 \\
\hline \multicolumn{3}{|l|}{ Distal metastasis } \\
\hline Yes & 57 & 43.2 \\
\hline No & 75 & 56.8 \\
\hline
\end{tabular}

F, female; M, Male.

\section{Study definition}

In the survival analysis, the primary end points were time until death, time until relapse, and time until metastasis. Overall survival (OS) was measured in days from the date of diagnosis to the date of death. Metastasis-free intervals were measured from the date of diagnosis to the date of metastasis (the metastatic location was either lung or bone) or death. The relapse-free interval was measured from the date of diagnosis to the 


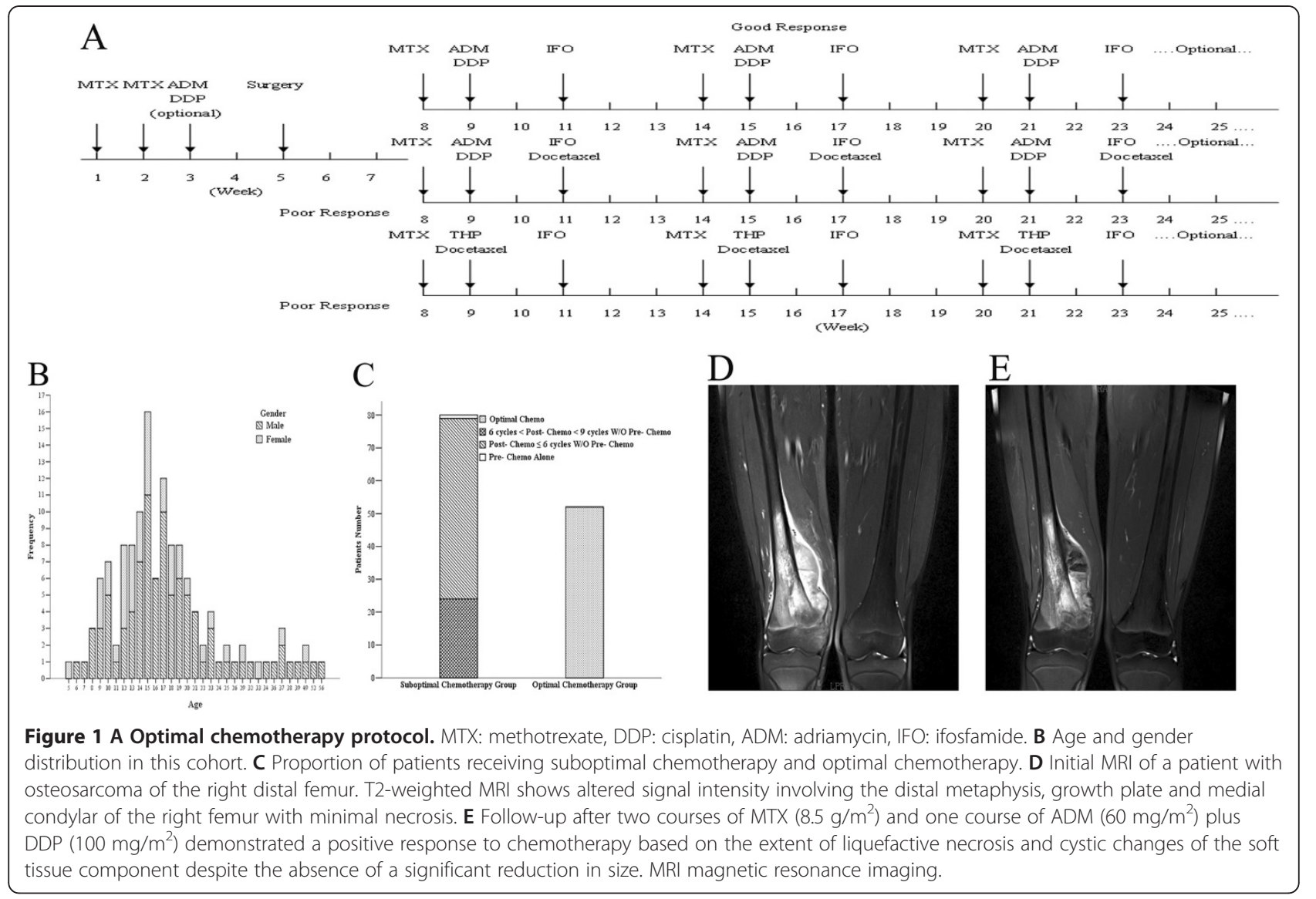

date of relapse or death. Event-free survival (EFS) was measured from the date of diagnosis to the date of any significant event (metastasis, relapse or death). Patients who did not develop metastasis or relapse or remained alive at the final follow-up were censored at that time.

\section{Surgery}

Surgical interventions for osteosarcoma included limb salvage surgery and amputation. Definitive surgical therapy required a wide or radical surgical margin. The options for postoperative reconstruction included modular prosthesis, extendible prosthesis, and autograft or allograft transplantation with plate or intramedullary interlocking nail. Amputation included simple amputation, rotationplasty amputation, and modified rotationplasty amputation. The surgical type was determined by the patient's life expectancy, maturity of epiphysis, presence of pathological fracture, clinical and surgical classification, and the patient's willingness and financial capacities.

\section{Follow-up}

After completing chemotherapy, patients were followed up for two years with quarterly radiographs of the operated limb. Computed tomography (CT) of the chest was performed every three months for two years. Thereafter, the same protocol was repeated every six months for up to five years and then annually for up to the tenth year after surgery. All of the patients were followed up for at least two years after diagnosis unless they were deceased.

\section{Statistics}

Both OS and EFS were determined based on the KaplanMeier Survival Analysis, and the results in the different groups were compared using the log-rank test. Only variables that were significantly different in the univariate study were entered into the multivariate analysis using the Cox proportional-hazards regression model. The analysis was performed using SPSS 13.0 and $P$-values of $<0.05$ were considered to be statistically significant.

\section{Results}

\section{Demographic information}

Among the total 410 patients, 132 met the inclusion criteria and their charts were reviewed for inclusion in the study group. The study population included 93 males and 39 females, with a median age of 16 years (range, 5 to 56 years); $34(25.8 \%)$ patients had undergone the onset of their growth spurt (12 to 14 (females), 13 to 15 
(males) (Figure 1B). The distal femur was the dominant location affected by osteosarcoma (50\%), and the majority of the pathological sub-classifications were the osteoblast subtype (Table 1). Fifty-three (39.4\%) cases underwent optimal chemotherapy while 80 (60.6\%) cases did not complete the prescribed chemotherapy protocol. Among these, 2 cases underwent only preoperative chemotherapy, 23 cases underwent fewer than 9 cycles and more than 6 cycles of postoperative chemotherapy with or without preoperative chemotherapy, and 55 cases underwent fewer than 6 cycles of postoperative chemotherapy with or without preoperative chemotherapy (Figure 1C). Sixty-three $(47.7 \%)$ events were recorded. Local recurrence occurred in 25 cases, including 3 cases of isolated local recurrence and 22 cases that eventually developed metastasis. Metastasis occurred in 57 cases, and the involved sites were the lung (55 cases), bone (1 case) and other sites ( 1 case).

\section{Suboptimal chemotherapy was correlated with adverse overall survival, event free survival, relapse free survival and metastasis free survival in univariate analysis}

The five-year OS and EFS statistics are summarized in Table 2. The OS for the entire cohort was $43.5 \pm 5.6 \%$. However, patients who adhered to the optimal protocol had a five-year survival rate of $65.3 \pm 7.3 \%$, which was significantly better than the $30.8 \pm 6.9 \%$ survival rate for the suboptimal chemotherapy group $(P=0.013)$. Similar results were obtained in EFS: $55.0 \pm 9.0 \%$ patients were alive without disease in the optimal chemotherapy group, but only $31.1 \pm 7.4 \%$ patients survived longer than five years without significant findings in the suboptimal chemotherapy group. Among the patients with metastases in the optimal chemotherapy group, $49.0 \pm$ $7.2 \%$ survived for five years, while $30.4 \pm 6.0 \%$ of those in the suboptimal chemotherapy group was still alive after five years of follow up. The outcome of patients with local recurrence in the optimal chemotherapy group was better than that of patients in the suboptimal chemotherapy group (five-year survival of $39.5 \pm 6.7 \%$ versus $24.7 \pm 6.1 \%$ ) (Table 3 ).

Suboptimal chemotherapy is an independent unfavorable factor for overall survival, event free survival and metastasis free survival

Based on the multivariate analysis, chemotherapy was found to be an independent risk factor for OS, EFS and metastasis free survival. The risk of mortality and metastasis was approximately 1.8 -fold higher in patients with suboptimal chemotherapy (hazard ratio $(\mathrm{HR})=1.804$, $95 \%$ confidence interval $(\mathrm{CI})=1.016$ to 3.204$)$. Patients in the optimal chemotherapy group were more likely to experience recurrence $(\mathrm{HR}=1.670,95 \% \mathrm{CI}=0.987$ to 2.825 ), although chemotherapy did not independently
Table 2 Overall survival and event-free survival

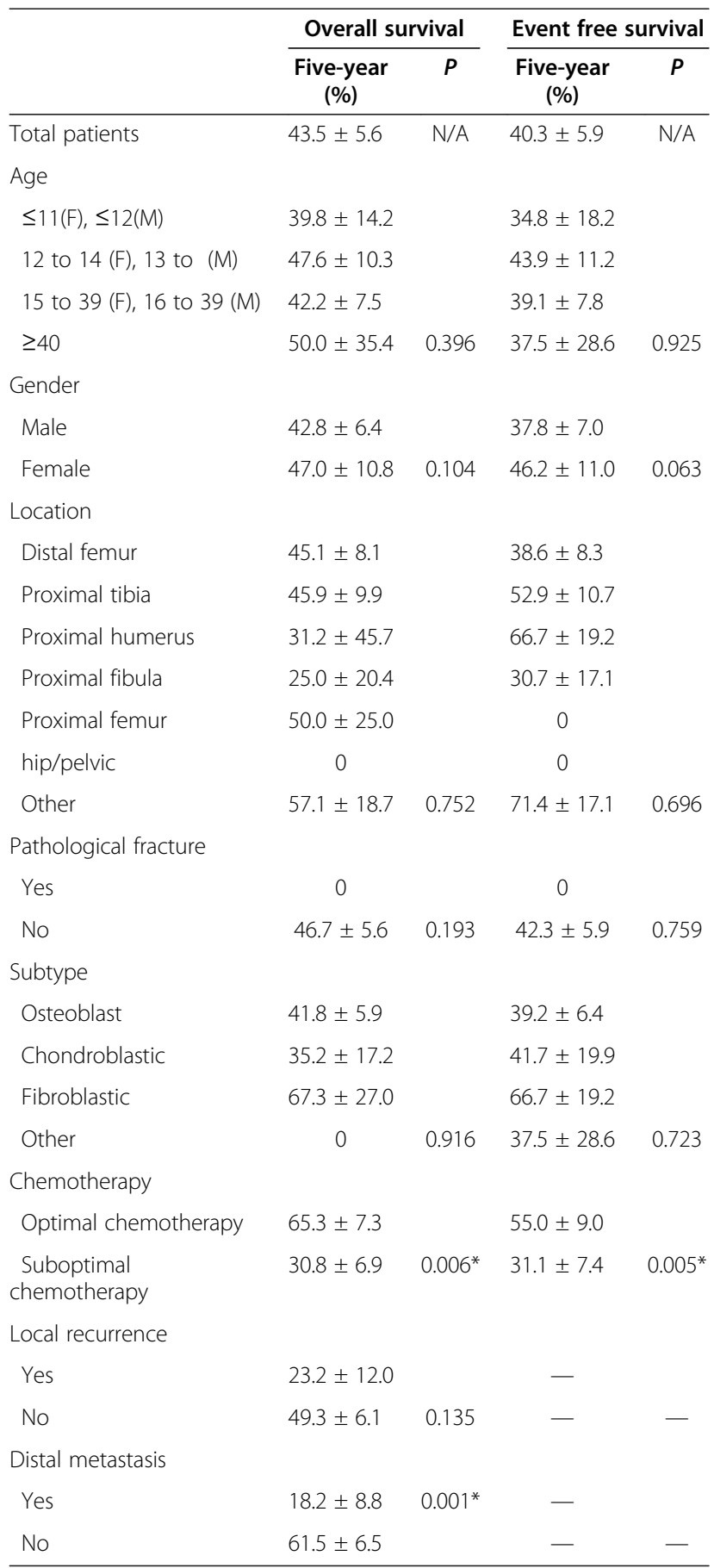

*, $P<0.05$; F, female; $M$, male.

affect relapse free survival $(P=0.06)$ (Table 4$)$. The Kaplan-Meier survival curves for the aforementioned data are shown in Figure 2.

\section{Discussion}

The addition of neoadjuvant and adjuvant chemotherapy to radical surgery has been demonstrated to improve 
Table 3 Relapse free survival and metastasis free survival

\begin{tabular}{|c|c|c|c|c|}
\hline & \multicolumn{2}{|c|}{$\begin{array}{c}\text { Relapse free } \\
\text { survival }\end{array}$} & \multicolumn{2}{|c|}{ Metastasis free survival } \\
\hline & Five-year & $P$ & Five-year & $P$ \\
\hline Total patients & $34.4 \pm 4.0$ & N/A & $33.6 \pm 3.9$ & N/A \\
\hline \multicolumn{5}{|l|}{ Age } \\
\hline$\leq 11(\mathrm{~F}), \leq 12(\mathrm{M})$ & $39.8 \pm 14.2$ & & $26.6 \pm 14.4$ & \\
\hline $12-14(F), 13-15(M)$ & $37.3 \pm 10.0$ & & $35.4 \pm 9.6$ & \\
\hline 15-39 (F), 16-39 (M) & $33.3 \pm 7.0$ & & $33.2 \pm 6.7$ & \\
\hline$\geq 40$ & $37.5 \pm 28.6$ & 0.941 & $37.5 \pm 28.6$ & 0.952 \\
\hline \multicolumn{5}{|l|}{ Gender } \\
\hline Male & $32.1 \pm 6.3$ & & $31.9 \pm 5.9$ & \\
\hline Female & $41.9 \pm 10.0$ & 0.090 & $38.0 \pm 9.7$ & 0.053 \\
\hline \multicolumn{5}{|l|}{ Location } \\
\hline Distal Femur & $37.3 \pm 7.7$ & & $32.4 \pm 7.2$ & \\
\hline Proximal Tibia & $45.9 \pm 9.9$ & & $41.8 \pm 9.4$ & \\
\hline Proximal humerus & $25.0 \pm 20.4$ & & $25.0 \pm 20.4$ & \\
\hline Proximal fibula & $30.7 \pm 17.1$ & & $46.0 \pm 17.5$ & \\
\hline Proximal Femur & $25.0 \pm 21.7$ & & 0 & \\
\hline Hip/Pelvic & 0 & & 0 & \\
\hline Others & 0 & 0.454 & $57.1 \pm 18.7$ & 0.257 \\
\hline \multicolumn{5}{|l|}{ Pathological Fracture } \\
\hline Yes & 0 & & 0 & \\
\hline No & $37.1 \pm 5.5$ & 0.319 & $35.5 \pm 5.2$ & 0.386 \\
\hline \multicolumn{5}{|l|}{ Subtype } \\
\hline Osteoblast & $33.5 \pm 5.7$ & & $31.9 \pm 5.4$ & \\
\hline Chondroblastic & $29.3 \pm 15.3$ & & $29.3 \pm 15.3$ & \\
\hline Fibroblastic & $66.7 \pm 19.2$ & & $83.3 \pm 15.2$ & \\
\hline Others & $45.0 \pm 32.2$ & 0.257 & $33.8 \pm 26.0$ & 0.326 \\
\hline \multicolumn{5}{|l|}{ Chemotherapy } \\
\hline Optimal Chemotherapy & $49.0 \pm 7.2$ & & $39.5 \pm 6.7$ & \\
\hline $\begin{array}{l}\text { Suboptimal } \\
\text { Chemotherapy }\end{array}$ & $30.4 \pm 6.0$ & $0.009^{*}$ & $24.7 \pm 6.1$ & $0.005^{*}$ \\
\hline \multicolumn{5}{|l|}{ Local Recurrence } \\
\hline Yes & - & & $13.7 \pm 8.0$ & \\
\hline No & - & - & $40.3 \pm 5.8$ & $0.033^{*}$ \\
\hline \multicolumn{5}{|l|}{ Distal Metastasis } \\
\hline Yes & $21.1 \pm 6.1$ & & - & \\
\hline No & $50.1 \pm 7.8$ & $0.001^{*}$ & - & - \\
\hline
\end{tabular}

*, $P<0.05$; F, female; $M$, male.

significantly the prognosis for osteosarcoma patients. Long-term survival in localized osteosarcoma has increased substantially from $10 \%$ to $20 \%$ (when surgery was performed as the only treatment) to $50 \%$ to $60 \%$ [12]. Five-year OS from 17 musculoskeletal oncology institutes in China ranges from $37.5 \%$ to $77.6 \%$ [13]. In the present study, the five-year OS for all patients was $43.5 \%$, which is significantly lower than the international survival rate for these patients [14-16]. However, the five-year OS among patients who completed the optimal chemotherapy regimen was as high as $65.3 \%$ which is comparable to the international survival rate. There is a suggestion that many patients failed to complete the optimal chemotherapy protocol, and this phenomenon is also observed at other Chinese hospitals.

A combination drug protocol consisting of four drugs (DDP, ADM, MTX, and IFO) has been associated with an overall five-year survival rate of $44 \%$ to $65 \%[17,18]$. International chemotherapy standards using these four drugs include the T12/T19 treatment developed by Rosen [19], the IOR-OS/N-2 treatment developed by Bacci and recommendations by the European Society for Medical Oncology (ESMO) in 2009 [20,21]. Although efforts have been made to identify optimized standard chemotherapy protocols that are suitable for the Chinese population, we do not yet have a nationwide sarcoma registry system that would support clinical trials with a large sample size to test standard protocols [8]. Our chemotherapy regimen was based on the four drugs described above and was a modified version of IOROS/N-2. The differences are as follows: (1) only two courses of MTX were required for induction chemotherapy and (2) IFO was routinely used after definitive surgery. For those who responded poorly to induction chemotherapy, we administered a more frequent IFO-containing regimen and/or added second-line drugs. The IOR-OS/N-2 protocol requires at least two cycles (four to six courses) for induction chemotherapy, but only two courses of preoperative medication are required in our protocol. We agree with Jeon and Bacci that the delayed removal of tumor increases the risk of systemic metastasis [22,23]. Furthermore, a smaller need exists for long-term neo-adjuvant therapy to bridge the gap between biopsy and resection due to the improvement of endoprostheses manufacturing. Third, the concern of non-compliance with long term induction chemotherapy must be considered. In our experience, two courses of MTX or two courses of MTX plus one course of $\mathrm{ADM}$ and DDP were adequate to achieve a good response to induction chemotherapy (Figure 1D and E). With this modified protocol, patients in the optimal chemotherapy group achieved an OS rate of $65.3 \% \pm 7.3 \%$, which is comparable to that in Bacci's study [19].

In the univariate study, factors that were significantly different according to our stratifications are chemotherapy, local recurrence and lung metastasis.

Bajpai et al. showed that there was no significant difference in survival between the noncompliant versus compliant group in osteosarcoma [24] but in our study, patients who failed to complete the optimal chemotherapy protocol had less opportunity to achieve a stable disease status or complete remission. The reason why these two studies have different conclusions is obvious. 
Table 4 Cox proportional hazards model for the risk of death, relapse or metastasis, relapse alone and metastasis alone

\begin{tabular}{|c|c|c|c|c|c|c|c|c|c|c|c|c|}
\hline & \multicolumn{3}{|c|}{ Overall survival } & \multicolumn{3}{|c|}{ Event free survival } & \multicolumn{3}{|c|}{ Relapse free survival } & \multicolumn{3}{|c|}{ Metastasis free survival } \\
\hline & HR & $95 \% \mathrm{Cl}$ & $P$ & HR & $95 \% \mathrm{Cl}$ & $P$ & HR & $95 \% \mathrm{Cl}$ & $\overline{P \text { value }}$ & HR & $95 \% \mathrm{Cl}$ & $P$ value \\
\hline \multicolumn{13}{|l|}{ Chemotherapy } \\
\hline Suboptimal chemo & 1.804 & 1.016 to 3.204 & & 2.512 & 1.242 to 3.729 & & 1.670 & 0.987 to 2.825 & & 1.863 & 1.159 to 2.996 & \\
\hline Optimal chemo & 1 & & 0.04 & & & $0.006^{*}$ & 1 & & 0.06 & 1 & & 0.01 \\
\hline \multicolumn{13}{|l|}{ Local recurrence } \\
\hline Yes & - & - & - & - & - & - & - & - & - & 0.633 & 0.388 to 1.034 & \\
\hline No & - & & & - & & & - & & & & & 0.07 \\
\hline \multicolumn{13}{|l|}{ Lung metastasis } \\
\hline Yes & 0.511 & $0.303-0.861$ & $0.01^{*}$ & - & - & - & 0.509 & $0.312-0.829$ & $0.007^{*}$ & - & - & - \\
\hline No & 1 & & & - & & & 1 & & & - & & \\
\hline
\end{tabular}

*, $\boldsymbol{P}<0.05 ; \mathrm{Cl}$,confidence interval; $\mathrm{HR}$, hazard ratio.

Bajpai's median follow up time is 7.9 months while our minimum follow-up time is 24 months. However, chemotherapy did not independently affect relapse-free survival $(P>0.05)$. These data indicate that other factors (such as surgery) act in combination with chemotherapy to maintain local control of the disease. Picci has convincingly demonstrated that the local failure rate in osteosarcoma correlates with both the quality of the surgical margins and the extent of tumor response to induction therapy [25]. Surgical margins should always be seriously considered to be equally as important as chemotherapy.

Chemotherapy compliance was defined as adherence to a prescribed treatment regimen [26]. It can be affected by psychological and behavioral aspects as well as financial aspects. The former may include physician's attitude, doctor-patient relationship, psychological support from others and religious convictions [27]. The latter was often unjustifiable and has social and economic roots. In China, the high price of drugs (including chemotherapy drugs and their rescue drugs) has long been blamed for medical services being unaffordable for less advantaged people and has triggered increasing complaints from the public [28]. Moreover, very expensive prosthesis (manufactured both domestically and abroad) causes some patients to discontinue chemotherapy after surgery. The current basic medical insurance system in China has not yet determined how to disseminate basic medical insurance coverage to benefit more patients with malignant tumors and how to reduce the proportion of personal health expenditures [29].

The possible solutions for unjustifiable chemotherapy non-compliance may include: (1) Increase the budget for medical services and restrain the price of chemotherapy drugs; (2) improve the national medical insurance system and enlarge the insurance coverage for malignant tumors; (3) encourage sponsorships and donations from society and set a charity fund for osteosarcoma patients; and (4) encourage domestic prosthesis development. The suggestions for justifiable non-compliance are as follow: (1) Strengthen the physician-patient relationship by providing adequate time for counseling, correct and accurate information interpretation, patient privacy protection and so on; (2) strengthen the family and social support system by providing personal and individualized care, chaplain services or psychological counseling; (3) strengthen patient education before and after hospitalization; and (4) strengthen the follow-up system by telephone, e-mail, and family visits to keep patients accountable for their timely therapy.

This study included some limitations. First, our study was limited by its retrospective nature; however, the issue under investigation could not be studied prospectively.

The prospective cohort study is of superior quality to a retrospective study in observational research; and the evidence level of a prospective study is higher than that of retrospective research. Nevertheless, it has the disadvantages of substantial expense, sensitivity to attrition and lengthy follow-up time. Desiring to improve our patients' survival rates, we conducted this study to investigate the effects of suboptimal chemotherapy on osteosarcoma prognosis.

Second, our study was devised using data available from osteosarcoma patients at a single institution, and therefore, the total number of patients was relatively small. Our results would be more compelling if a larger number of patients being treated at different institutions had been included in the study, but our strict standards for inclusion naturally limited the size of our study. Our results also require validation in a large number of patients at different institutions. Finally, bias led by the inherent heterogeneity of the optimal chemotherapy regimen is difficult to eliminate.

\section{Conclusions}

In summary, we investigated a cohort of osteosarcoma patients with long term follow-up, suboptimal chemotherapy compromised the outcome and 

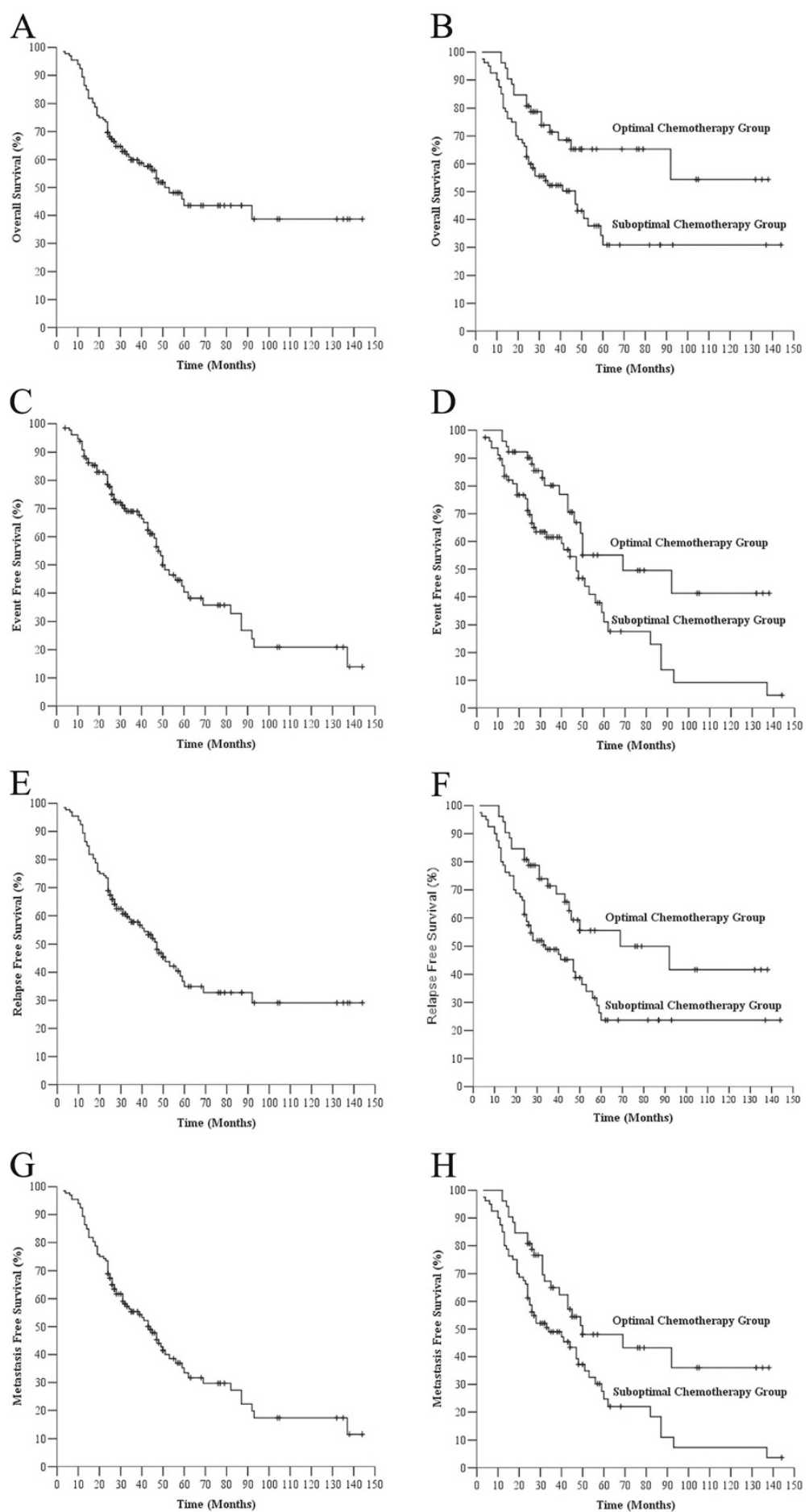

Figure 2 A Overall survival for all patients, compared to B Overall survival stratified by chemotherapy. C Event-free survival for all patients, compared to $\mathbf{D}$ Event-free survival stratified by chemotherapy. E Relapse-free survival for all patients, compared to $\mathbf{F}$ Relapse-free survival stratified by chemotherapy. G Metastasis-free survival for all patients, compared to $\mathbf{H}$ Metastasis-free survival stratified by chemotherapy. 
prognosis of the subjects. Standardization of treatment is deemed necessary in the Chinese osteosarcoma population, and multicenter clinical trials to identify optimized chemotherapy drugs and protocols suitable for Chinese patients must be developed. Moreover, taking measures to improve patients' compliance to treatment is of equal importance.

\section{Abbreviations}

ADM: adriamycin; Cl: confidence interval; DDP: cisplatin; EFS: eventfree survival; ESMO: European Society for Medical Oncology; HR: hazard ratio; IFO: ifosfamide; MTX: methotrexate; OS: overall survival.

\section{Competing interests}

We declare that we have no conflicts of interest.

\section{Authors' contributions}

JNS and JW conceived the study. BCY, PXT, CYZ did the chart review. BCY and YQW followed up the patients and obtained the survival information. $\mathrm{GH}$ and $\mathrm{BCY}$ performed the statistical analysis and interpreted the results. $B C Y, X B X$ and JQY performed the literature review and wrote the manuscript. All authors read and approved the final manuscript.

\section{Authors' information}

Professor JNS is the director and chief surgeon of Musculoskeletal Department of First Affiliated Hospital of Sun Yat-Sen University, China.

\section{Acknowledgements}

We thank Dr. Yaofei Wang, Dr. Jingchun Li, Dr. Jinchang Lu, and Dr. Haihua Guo for their efforts in helping gather the data. We are also grateful to Zhibin Li for his assistance with the statistical analysis. We also give credit to Patricia Yong for her critical editing of the manuscript. This study was supported by Sun Yat-Sen University 5010 Project (grant number: 180009).

Received: 3 May 2012 Accepted: 31 August 2012

Published: 17 September 2012

\section{References}

1. Bielack SS, Kempf-Bielack B, Delling G, Exner GU, Flege S, Helmke K, Kotz R, Salzer-Kuntschik M, Werner M, Winkelmann W, Zoubek A, Jürgens $H$, Winkler K: Prognostic factors in high-grade osteosarcoma of the extremities or trunk: an analysis of 1,702 patients treated on neoadjuvant cooperative osteosarcoma study group protocols. J Clin Oncol 2002, 20:776-790.

2. Savage SA, Mirabello L: Using epidemiology and genomics to understand osteosarcoma etiology. Sarcoma 2011, 2011:548151.

3. Chou AJ, Gorlick R: Chemotherapy resistance in osteosarcoma: current challenges and future directions. Expert Rev Anticancer Ther 2006, 6:1075-1085.

4. Fuchs N, Bielack SS, Epler D, Bieling P, Delling G, Korholz D, Graf N, Heise U, Jurgens H, Kotz R, Salzer-Kuntschik M, Weinel P, Werner M, Winkler K: Long-term results of the co-operative German-Austrian-Swiss osteosarcoma study group's protocol COSS-86 of intensive multidrug chemotherapy and surgery for osteosarcoma of the limbs. Ann Oncol 1998, 9:893-899.

5. Bacci G, Ferrari S, Longhi A, Picci P, Mercuri M, Alvegard TA, Saeter G, Donati D, Manfrini M, Lari S, Briccoli A, Forni C, Italian Sarcoma Group/Scandinavian Sarcoma Group: High dose ifosfamide in combination with high dose methotrexate, adriamycin and cisplatin in the neoadjuvant treatment of extremity osteosarcoma: preliminary results of an Italian Sarcoma Group/Scandinavian Sarcoma Group pilot study. J Chemother 2002, 14:198-206.

6. Ferrari S, Smeland S, Mercuri M, Bertoni F, Longhi A, Ruggieri P, Alvegard TA, Picci P, Capanna R, Bernini G, Müller C, Tienghi A, Wiebe T, Comandone A, Böhling T, Del Prever AB, Brosjö O, Bacci G, Saeter G, Italian and Scandinavian Sarcoma Groups: Neoadjuvant chemotherapy with highdose Ifosfamide, high-dose methotrexate, cisplatin, and doxorubicin for patients with localized osteosarcoma of the extremity: a joint study by the Italian and Scandinavian Sarcoma Groups. J Clin Onco/ 2005, 23:8845-8852.

7. Meyers PA, Schwartz CL, Krailo MD, Healey JH, Bernstein ML, Betcher D, Ferguson WS, Gebhardt MC, Goorin AM, Harris M, Kleinerman E, Link MP, Nadel H, Nieder M, Siegal GP, Weiner MA, Wells RJ, Womer RB, Grier $H E$, Children's Oncology Group: Osteosarcoma: the addition of muramyl tripeptide to chemotherapy improves overall survival--a report from the Children's Oncology Group. J Clin Oncol 2008, 26:633-638.

8. Lin F, Wang Q, Yu W, Tang L, Zheng S, Sun Y, Shen Z, Yao Y, Dong Y: Clinical analysis of Chinese limb osteosarcoma patients treated by two combinations of methotrexate, cisplatin, doxorubicin and ifosfamide. Asia Pac J Clin Oncol 2011, 7:270-275.

9. Enneking WF, Spanier SS, Goodman MA: A system for the surgical staging of musculoskeletal sarcoma. Clin Orthop Relat Res 1980, (153):106-120

10. Uchida A, Myoui A, Araki N, Yoshikawa H, Shinto Y, Ueda T: Neoadjuvant chemotherapy for pediatric osteosarcoma patients. Cancer 1997, 79:411-415.

11. Therasse P, Arbuck SG, Eisenhauer EA, Wanders J, Kaplan RS, Rubinstein L, Verweij J, Van Glabbeke M, van Oosterom AT, Christian MC, Gwyther SG: New guidelines to evaluate the response to treatment in solid tumors. European Organization for Research and Treatment of Cancer, National Cancer Institute the United States, National Cancer Institute of Canada. J Natl Cancer Inst 2000, 92(3):205-216.

12. Pazdur R, Wagman LD, Camphausen KA, Hoskins WJ: Cancer Management: A Multidisciplinary Approach. 11th edition. Manhaset, NY: CMP Medica; 2009.

13. Zhang Q, Xu WP, Guo W: The current status of the treatment for osteosarcoma in China. Chin J Bone Tumor Bone Dis 2009, 8:129-132.

14. Smeland S, Bruland OS, Hjorth L, Brosjo O, Bjerkehagen B, Osterlundh G, Jakobson A, Hall KS, Monge OR, Bjork O, Alvegaard TA: Results of the Scandinavian Sarcoma Group XIV protocol for classical osteosarcoma: 63 patients with a minimum follow-up of 4 years. Acta Orthop 2011, 82:211-216.

15. Gatta G, Zigon G, Capocaccia R, Coebergh JW, Desandes E, Kaatsch P, Pastore G, Peris-Bonet R, Stiller CA: Survival of European children and young adults with cancer diagnosed 1995-2002. Eur J Cancer 2009, 45:992-1005.

16. Bielack $S$, Jurgens $H$, Jundt $G$, Kevric M, Kuhne $T$, Reichardt $P$, Zoubek $A$, Werner M, Winkelmann W, Kotz R: Osteosarcoma: the COSS experience. Cancer Treat Res 2009, 152:289-308.

17. Longhi A, Errani C, De Paolis M, Mercuri M, Bacci G: Primary bone osteosarcoma in the pediatric age: state of the art. Cancer Treat Rev 2006, 32:423-436

18. Ferrari S, Palmerini E: Adjuvant and neoadjuvant combination chemotherapy for osteogenic sarcoma. Curr Opin Oncol 2007, 19:341-346.

19. Rosen G, Caparros B, Huvos AG, Kosloff C, Nirenberg A, Cacavio A, Marcove RC, Lane JM, Mehta B, Urban C: Preoperative chemotherapy for osteogenic sarcoma: selection of postoperative adjuvant chemotherapy based on the response of the primary tumor to preoperative chemotherapy. Cancer 1982, 49:1221-1230.

20. Bacci G, Longhi A, Fagioli F, Briccoli A, Versari M, Picci P: Adjuvant and neoadjuvant chemotherapy for osteosarcoma of the extremities: 27 year experience at Rizzoli Institute, Italy. Eur J Cancer 2005, 41:2836-2845.

21. Bielack S, Carrle D, Casali PG: Osteosarcoma: ESMO clinical recommendations for diagnosis, treatment and follow-up. Ann Oncol 2009, 20(Suppl 4):137-139.

22. Jeon DG, Song WS: How can survival be improved in localized osteosarcoma? Expert Rev Anticancer Ther 2010, 10:1313-1325.

23. Bacci G, Ferrari S, Longhi A, Forni C, Ruggieri P, Briccoli A, De Paolis M, Setola E: Preoperative therapy versus immediate surgery in nonmetastatic osteosarcoma. J Clin Oncol 2003, 21:4662-4663.

24. Bajpai J, Puri A, Shah K, Susan D, Jambhekar N, Rekhi B, Desai S, Gulia A, Gupta S: Chemotherapy compliance in patients with osteosarcoma. Pediatr Blood Cancer 2012, doi:10.1002/pbc.24155.

25. Picci P, Sangiorgi L, Rougraff BT, Neff JR, Casadei R, Campanacci M: Relationship of chemotherapy-induced necrosis and surgical margins to local recurrence in osteosarcoma. J Clin Oncol 1994, 12:2699-2705.

26. Lewis C, Linet MS, Abeloff MD: Compliance with cancer therapy by patients and physicians. Am J Med 1977, 101:14-18. 
27. Balasubramanian SP, Murrow S, Holt S, Manifold IH, Reed MW: Audit of compliance to adjuvant chemotherapy and radiotherapy guidelines in breast cancer in a cancer network. Breast 2003, 12:136-141.

28. Yu X, Li C, Shi Y, Yu M: Pharmaceutical supply chain in China: current issues and implications for health system reform. Health Policy 2010, 97:8-15.

29. Song $\mathrm{P}, \mathrm{Wu} \mathrm{Q}$, Huang $\mathrm{Y}$ : Multidisciplinary team and team oncology medicine research and development in China. Biosci Trends 2010, 4:151-160.

doi:10.1186/1477-7819-10-191

Cite this article as: Yong et al:: Suboptimal chemotherapy is an adverse prognostic factor in osteosarcoma. World Journal of Surgical Oncology 2012 10:191.

\section{Submit your next manuscript to BioMed Central and take full advantage of:}

- Convenient online submission

- Thorough peer review

- No space constraints or color figure charges

- Immediate publication on acceptance

- Inclusion in PubMed, CAS, Scopus and Google Scholar

- Research which is freely available for redistribution 